\title{
Ärztin, 30, Krise
}

\section{Mirjam Tanner}

Dr. med., Fachärztin für Psychiatrie und Psychotherapie, Leitungsausschuss ReMed

Es ist die häufigste Geschichte, die das Unterstützungsnetzwerk ReMed auf ähnliche Weise immer wieder neu zu hören bekommt und hier exemplarisch erzählt wird: Kompetente, sensible und enorm engagierte Assistenzärztinnen mit hohen Idealen sind so frustriert von ihrem Arbeitsalltag, dass sie sich - und ihre Berufswahl - völlig hinterfragen. Die Autorin rät dringend, junge Kolleginnen bewusst zu unterstützen, um sie während der Weiterbildung nicht zu verlieren.

"Als ich mich an ReMed wende, glaube ich, entweder im nächsten Moment auseinanderzufallen oder aber von einem Monster aufgefressen zu werden. Mein Herz rast, der Schweiss klebt das T-Shirt auf mir fest und mir ist schwindlig. Die warme Stimme des ReMedBeraters am Telefon beruhigt mich etwas, so dass ich ihm meine Situation schildern kann. Ich bin 32, am Schluss meiner Ausbildung zur Internistin, habe die letzte Stelle vor zwei Monaten angetreten - und kann nicht mehr. Es ist alles nur noch eine Tortur, die Anspannung, die Ängste, der Stress, die Vorwürfe wegen meiner Überstunden, das macht alles keinen Sinn mehr. Ich bewundere die anderen, die alles so locker schaffen. Ich aber scheine unfähig für diesen Beruf und bin enttäuscht.

Es war schon mein Kindheitstraum, Ärztin zu werden, einmal Menschen zu helfen und Leben zu retten, vor allem das von Kindern, um die sich niemand kümmert. Inzwischen bin ich zwar Ärztin, aber eine, die durch ihren Stress im beruflichen Alltag keine Zeit findet, um auf ihre Patienten wirklich einzugehen und ihnen richtig zuzuhören. Es kommt mir jeweils vor, also ob immer mindestens tausend Leute irgendetwas von mir wollen und zwar sofort. Ich aber bin von Natur aus langsam, sorgfältig und nicht multitaskingfähig.

\section{Erschöpft, desillusioniert, verunsichert}

Gegenwärtig bin ich mit den Nerven oft derart am Ende, dass ich ungeduldig, unfreundlich und sehr distanziert werde. Ich merke, dass ich manchmal Patienten brüskiere. Was ist aus meinem Kindheitstraum bloss geworden? Einer meiner Leitenden Ärzte hat mir gesagt, dass ich zu hohe Ideale hätte und meine Arbeit viel zu genau machen wolle. Ich müsse lernen, mich abzugrenzen und nicht alles noch einmal zu kontrollieren und zu hinterfragen, sondern mit Sicherheit

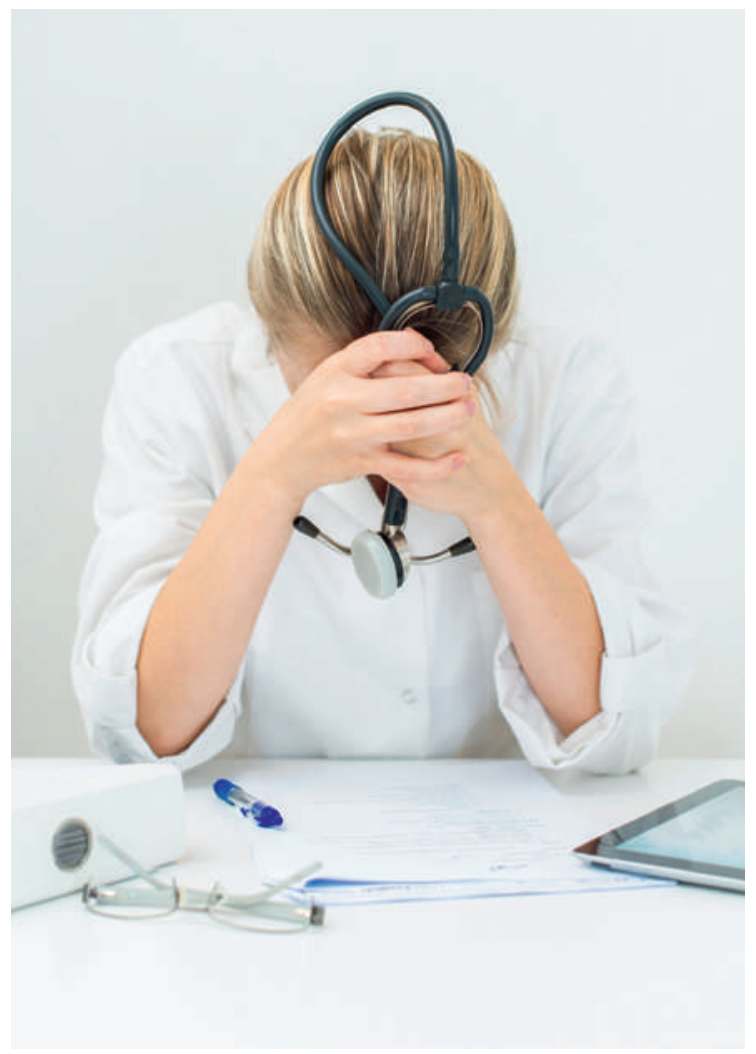

Am Ende der Kräfte und ohne Perspektiven statt Freude am Beruf und ein erfülltes Leben? Hier kann ReMed Orientierung und neuen Mut geben.

\section{ReMed ist für Sie da}

Benötigen Sie oder jemand aus Ihrem Umfeld professionelle Hilfe? Wenden Sie sich an ReMed: Das Unterstützungsnetzwerk für Ärztinnen und Ärzte respektiert das Arztgeheimnis und berät Sie kompetent. ReMed begleitet Sie in beruflichen und persönlichen Krisen. Auf Ihre Kontaktnahme meldet sich das Beraterteam innerhalb von 72 Stunden zurück. Weitere Informationen und Kontakt: www.swiss-remed.ch, help[at] swiss-remed.ch, Tel. 0800073633. 
Entscheide zu fällen. Doch seit eine meiner Patientinnen an einer Lungenembolie gestorben ist, die auch die Oberärztin nicht diagnostiziert hatte, kämpfe ich jeden Tag gegen meine Angst, etwas zu übersehen. Jeden Morgen, wenn ich durch den Haupteingang der Klinik trete, überkommen mich Selbstzweifel und die zermürbende Überzeugung, dass alle wissen, dass ich eine untaugliche Ärztin bin. Nicht, dass der Chef das jemals erwähnt hätte. Ich verstehe nicht, warum er so grosses Vertrauen in meine Fähigkeiten hat und sogar eine Versetzung auf seine Privatabteilung vorschlug. Es ist hart, wenn ich das so sage: Mir kommt es vor, als beweise er damit seine eigene Inkompetenz.

\section{Einsamkeit, Trauer, Torschlusspanik}

Was für soziale Kontakte ich pflege, fragt der Herr von ReMed. Lange sage ich nichts, bemerke stattdessen, wie mir Wärme im Kopf aufschiesst und Tränen die Wangen herunterlaufen. Es ist eine ernüchternde Frage. Das letzte Mal verabredet war ich vor etwa zwei Monaten mit meiner Schwester. Eine Freundin in einer

\section{Ich kämpfe jeden Tag gegen meine Angst, etwas zu übersehen.}

anderen Stadt habe ich noch, mit der ich gelegentlich telefoniere. Seit ich als Assistenzärztin arbeite, mache ich nicht mehr ernsthaft Musik. Früher sang ich in einer Band, jedoch konnte ich mit den unregelmässigen Arbeitszeiten nicht mehr genügend proben. Und eigentlich bin ich auch als Sängerin immer unzufrieden mit mir gewesen. Ich habe nicht nur im Beruf, sondern in allem sehr hohe Ansprüche an mich.

Ich bin kompliziert, und das belastet auch meine Männerbeziehungen. Eine riesige Sehnsucht und Traurigkeit stecken in mir. Ich schäme mich, dass ich seit vier Jahren Single bin, und für die jährlich steigende Torschlusspanik in Bezug auf meinen Kinderwunsch. So kann man ja unmöglich den passenden Partner finden! Diesem Lebensbereich gegenüber fühle ich mich ohnmächtig. Wie bestraft, nicht liebenswert und nicht attraktiv genug für die Männer, die mich interessieren würden. Die letzte Partnerschaft hatte ich mit einem Kollegen, den ich fachlich sehr bewundere. Wir waren drei Jahre zusammen. Er beendete schliesslich die Beziehung, weil wir so verschiedene Vorstellungen vom Leben hätten. Er ist inzwischen Vater und mit einer Krankenschwester verheiratet, die wegen der beiden Kinder aufgehört hat zu arbeiten.

\section{Sich neu wahrnehmen und ausrichten}

Selbst wenn ich täglich mit Gedanken spiele, meinen Beruf an den Nagel zu hängen und etwas ganz anderes zu tun, weiss ich genau, dass mich dies langfristig kein bisschen glücklicher machen würde. Ich frage den ReMed-Berater, wie ich meine verlorene Stärke wieder finden könne. Wir kristallisierten heraus, dass ich von aussen betrachtet im Beruf gute Arbeit leiste, mir selbst jedoch nie genüge. Ich stehe unter realem Zeitdruck. Aber der grösste Teil des restlichen Druckes, unter dem ich mich fühle und der oft in Panik ausartet, scheine ich mir selbst zu machen. Irgendwann kann das innere System einfach kein Gegensteuer mehr geben und setzt aus. Auch meine freudlose persönliche Lebenssituation blockiert, schwächt und stresst mich permanent.

Ich weiss noch nicht, wie ich mein Leben dahingehend verändern kann, dass es mich wieder erfüllt. Zumindest haben mir die zwei Gespräche mit dem ReMedBerater geholfen, mir meine Sehnsucht nach einer Partnerschaft und auch meinen Kinderwunsch offen einzugestehen und als einen wichtigen und wertvollen Teil meiner Selbst zu betrachten. Wir diskutieren verschiedene mögliche unterstützende Angebote für mich, u.a. ein Tutoring, ein Coaching oder eine Psychotherapie. Noch habe ich mich nicht entschieden und bin sehr ambivalent, ob ich weitere Hilfe beanspruchen möchte. Dank des Kontakts mit ReMed habe ich bereits etwas Ruhe und Orientierung gewonnen. Es stärkt mich auch zu wissen, dass ich mich ohne Scham oder Zögern wieder an den ReMed-Berater wenden kann, wenn ich meinen Problemen doch mit professioneller Unterstützung auf den Grund gehen möchte. Er wird mir helfen, eine erfahrene und geeignete Person dafür zu finden.»

Bildnachweis

(C) Dmitri Maruta | Dreamstime.com

\section{Intervisionsgruppen: Termine 2015}

ReMed initiierte 2009 kollegiale Intervisionen, auch auf Wunsch von Kolleginnen und Kollegen. Seither organisiert das Unterstützungsnetzwerk regelmässig Peer-Groups (6-10 Teilnehmer, 2-3-mal/Jahr). Die Teilnehmenden erarbeiten gemeinsam ihre Fallfragen zu Mentoring, Coaching, Beratung, Therapie und anderen Aspekten kollegialer Begleitung (juristisch, versicherungsrechtlich usw.). Setzen Sie sich mit uns in Verbindung, nehmen Sie an einer Sitzung teil und lernen Sie unsere Arbeit kennen. Kontakt: Peter Birchler, Tel. 0443420910 oder peter. birchler[at]hin.ch

Restliche Daten für 2015: 12.11. Hotel Central Plaza, Zürich; 19.11. Hotel Central Plaza, Zürich 